\title{
Frühkindliche Ernährung: Einfluss auf Entwicklung und Gesundheit
}

_ Im Kleinkindalter ist Milch ein entscheidender Lieferant für Kalzium, Jod, Vitamin B2, Vitamin D sowie für Proteine. Das Forschungsinstitut für Kinderernährung (FKE) empfiehlt im Rahmen einer optimierten Mischkost den Verzehr von ca. einem Drittel Liter fettarmer Milch pro Tag, was in etwa einer täglichen Proteinzufuhr von $1 \mathrm{~g} / \mathrm{kg}$ KG am Tag entspricht. Untersuchungen zeigen jedoch, dass die tatsächliche tägliche Proteinzufuhr mit ca. 2,5 g/kg $\mathrm{KG} /$ Tag deutlich über den empfohlenen Werten liegt und dass ein erhöhter Proteinverzehr im zweiten Lebensjahr ein er- höhtes Risiko für einen zu hohen BMI und Körperfettgehalt im Kindesalter mit sich bringt [Am J Clin Nutr 2007; 86: 1765-72]. Professor Dr. Mathilde Kersting, Dortmund, verwies deshalb auf aktuelle Ergebnisse der seit 1985 andauernden DONALD-Studie (Dortmund Nutritional and Anthropometric Longitudinally Designed), denen zufolge die tägliche Proteinzufuhr durch Verwendung proteinreduzierter Kindermilchprodukte, wie z.B. Aptamil ${ }^{\circledR}$ Kinder-Milch, verringert werden kann.

Ein entscheidender Faktor für eine gesunde, abwechslungsreiche Ernährung ist der individuelle Geschmack. PD Dr. Walter Mihatsch, Schwäbisch Hall, erklärte, dass die Geschmacksprägung bereits im Mutterleib beginne und sich im frühkindlichen Alter fortsetze, weshalb bereits früh auf eine vielfältige, ausgewogene Kost geachtet werden sollte. Besonders auf die Verwendung von Zucker- und Aromastoffen sollte bei Kleinkindnahrung verzichtet werden, da sich die daraus entstehenden Präferenzen bis ins Erwachsenenalter fortsetzen und beispielsweise zu einer sehr zuckerreichen Ernährung führen können.

Industriesymposium „Programmiert die frühkindliche Ernährung die langfristige Gesundheit?" anlässlich der 106. Jahrestagung der DGKJ. Potsdam, 17. September 2010. Veranstalter: Milupa, Friedrichsdorf

\section{Comeback für Lachgas}

- Das geschmack-, farb- und geruchlose Gas Distickstoffmonoxid, bekannter unter dem Begriff „Lachgas“, das seinen Namen aufgrund seiner psychotropen Effekte erhielt, zieht nach langem Schattendasein wieder in bundesdeutsche Praxen ein. Indikationen für LIVOPAN ${ }^{\circledR}$, das seit August 2008 zugelassenen Fertigarzneimittel in Form eines Gasgemischs aus 50\% Distickstoffmonoxid und 50\% Sauerstoff, sind kurze Eingriffe mit Schmerzzuständen von leichter bis mittelstarker Intensität. In Deutschland wird das schnell an- und abflutende Schmerzmittel bei Kindern vor allem für Lumbal- bzw. Gelenkpunktionen eingesetzt. Aber auch zur Metallentfernung, für Verbandwechsel und Wundversorgung eignet sich das inhalative Analgetikum. „Die Anwendung des Lachgas-Sauerstoffgemischs ist ein sicheres, komplikationsarmes Verfahren, das auch von NichtAnästhesisten durchgeführt werden kann", erklärte PD Dr. Stefan Holland-Cunz, Heidelberg. Er betonte jedoch, dass das Personal geschult sein sollte.

Im Rahmen der 106. Jahrestagung der Deutschen Gesellschaft für Kinder- und Jugendmedizin in Potsdam konnten die ersten Erfahrungen, die in Kinderkliniken und Kindernotaufnahmen mit LIVOPAN ${ }^{\circledR}$ gemacht wurden, vorgestellt werden. Die Referenten waren sich darin einig, dass die bisherigen Behandlungsergebnisse bei Kindern über drei Jahre durchweg positiv sind. Um vergleichbare Daten in größerer Menge für valide Aussagen zu generieren, müsste jedoch die Anwendung in Deutschland weiter standardisiert werden. Zudem wird LIVOPAN ${ }^{\circledR}$ in Deutschland noch zu wenig eingesetzt, bemängelte Mathias Genné aus Cottbus, obwohl sein Einsatz sehr gut steuerbar und nur mit einer kurzen Vor- und Nachbeobachtungszeit verbunden sei.

CVS

Industriesymposium „LIVOPAN ${ }^{\circledR}$ - inhalative Analgesie für kurze, schmerzhafte Eingriffe bei Kindern" anlässlich der 106. Jahrestagung der DGKJ. Potsdam, 18. September 2010. Veranstalter: Linde Gas Therapeutics, Unterschleißheim

\section{Rotavirus-Infektionen: ein vermeidbares Risiko}

Fast jedes Kind muss sich im Laufe seiner ersten zwei bis drei Lebensjahre mindestens einer Infektion mit dem Rotavirus (RV) stellen. Warum damit nicht zu spaßen ist, erklärte Prof. Dr. Hans-Iko Huppertz aus Bremen: Die durch RV hervorgerufene akute Gastroenteritis verläuft meist schwerer als die durch andere Erreger verursachte. Es droht die schwere hypertone Dehydratation. Und selbst wenn dem Kind die Einweisung ins Krankenhaus erspart bleibt, deutet eine Studie auf eine unerwartet hohe Beeinträchtigung der Familie während der Erkrankung hin.

Die Effektivität einer generellen Schutzimpfung gegen RV zeigt das Beispiel Sachsen. Das Bundesland hat die RV-Impfung bereits Anfang 2008 öffentlich empfohlen, entsprechend stiegen die Impfraten auf 38\% im Jahr 2008 und $57 \%$ im Jahr 2009. Die Folge: Die Morbidität bei den Säuglingen sank in diesen Jahren um 21 bzw. 50\%. Bei den Ein- bis Zweijährigen zeigte sich 2009 eine Morbiditätssenkung um $20 \%$.

Im Hinblick auf die oft unterschätzte Bedeutung einer RV-Infektion sei eine möglichst frühe Aufklärung der Eltern über die Gefahr und die schützende Impfung (z.B. mit Rotarix ${ }^{\circledast}$ ) umso wichtiger, so Huppertz. Das Problem der fehlenden generellen Kostenübernahme durch die Krankenkassen wird dabei kleiner: Immer mehr Kassen erstatten die Impfung gegen RV. Eine aktuelle Liste ist online unter www.gesundes-kind.de zu finden.

bs

Impfsymposium „Rotavirusimpfung - was gibt es Neues?" anlässlich der 106. Jahrestagung der DGKJ. Potsdam, 18. September 2010. Veranstalter: GlaxoSmithKline, München 